\title{
Effect of Broomrape (Orobanche spp.) Infection on Sugar Content of Carrot Roots
}

\author{
A.A. Schaffer ${ }^{1}$ R. Jacobsohn, D.M. Joel ${ }^{2}$, E. Eliassi, and \\ Miriam Fogelman \\ Agricultural Research Organization, Institute of Field and Garden Crops, \\ Department of Vegetable Crops, P.O.B. 6, Bet Dagan 50250, Israel
}

Additional index words. Daucus carota

Abstract. The soluble sugar content of mature carrot roots (Daucus carota L. CV. TiTo, Nantes type) grown in broomrape-infested or noninfested soil was measured. Orobanche aegyptiaca Pers. (Egyptian broomrape) and O. crenata Forssk. (crenate broomrape) decreased the total sugar content similarly. Sucrose was the primary sugar in noninfected roots and its level was greatly reduced in infected roots. Concentrations of sucrose were higher in the cortex than in the core; broomrape infection reduced sucrose concentration in both portions of the root. Even broomrape-infected roots with a healthy appearance (acceptable size and color) had a greatly reduced sucrose content. These results show that broomrape infection can reduce carrot root quality even when the visual appearance of the root is not affected.

Carrot quality is determined in part by its sugar content (Simon et al., 1980). Phan and Hsu (1973) have reported that the sugar content in developing carrot roots is due primarily to the accumulation of sucrose, whereas reducing sugar concentrations do not change significantly throughout development. However, some carrot genotypes accumulate reducing sugars, rather than sucrose (Freeman and Simon, 1983).

Broomrape is a root holoparasitic flowering plant without chlorophyll that attacks the roots of various crops such as tomato, legumes, and sunflower (Joel, 1987; Musselman, 1980) and causes considerable damage to agricultural crops throughout the world (Musselman, 1986; Parker, 1986). It causes major losses in carrot yields and quality (Jacobsohn et al., 1980). In heavily infested fields, many of the roots are small and misshapen, with poor color. However, many other roots appear normal with respect to size and color, although parasitized by Orobanche plants.

Whitney (1972) and Aber et al. (1983) reported that sucrose is the primary sugar withdrawn from faba bean plants by parasitizing Orobanche crenata. Sucrose is subsequently hydrolyzed to glucose and fructose by the parasite (Whitney, 1972), which in turn accumulates large amounts of starch (Singh et al., 1968). The purpose of our study

Received for publication 6 Aug. 1990. Contribution from the Agricultural Research Organization, The Volcani Center, Bet Dagan 50250, Israel, no. 2946-E, 1990 Series. The cost of publishing this paper was defrayed in part by the payment of page charges. Under postal regulations, this paper therefore must be hereby marked advertisement solely to indicate this fact.

'Author to whom correspondence should be addressed.

${ }^{2}$ Dept. of Weed Research, Agricultural Research Organization, Newe Ya'ar Experiment Station, P.O. Haifa, 31999, Israel. plot was planted with several crops, including carrot, as part of a study to determine the sensitivity of crops to different broomrape species.

Carrots cv. Ti-To, a Nantes type, were seeded on 28 Oct. 1988, in 1.6-m-wide raised beds. Rows were sidedressed with $190 \mathrm{~kg} \mathrm{~N}$ and $100 \mathrm{~kg} \mathrm{P} / \mathrm{ha}$. By Spring 1989, carrots were heavily parasitized in the plots inoculated with $O$. crenata Forsk (> 100 O. crenata spikes $/ \mathrm{m}$ ) and in those inoculated with O. aegptiaca Pers. (> 20 spikes $/ \mathrm{m}$ ). At the same time, carrots were not infested in the $O$. cumana and $O$. cernua plots. Therefore, carrot from the latter two plots were used as uninfected controls.

Carrot roots were harvested, washed, graded visually, and checked individually for broomrape infection. Cross-sectional samples from the midportion of the root were immediately placed in $80 \%$ ethanol (EtOH) and stored at - 20C until analysis. In expts. 1 and 2, the cross sections were separated into core and cortex, which were analyzed for sucrose separately.

Soluble sugars were extracted in hot $80 \%$ EtOH $(3 \times)$ and sucrose, glucose, and fructose were assayed by high performance liquid chromatography using a Bio-Rad Fast Carbohydrate Analysis column (Bio-Rad, Richmond, Calif.) with double-distilled $\mathrm{H}_{2} \mathrm{O}$ as solvent, and refractometric detection, according to manufacturer's directions. Starch was measured as previously reported (Schaffer et al., 1987).

Three experiments were conducted with different harvest dates during April-May 1989:

Expt. 1. Roots were harvested on 19 Apr. 1989 from noninfested plots and from plots infested with either $O$. aegyptiaca or $O$. crenata. Roots that showed attachment of broomrape were separated from uninfected rows. Rate of carrot seeding was 100 seeds/ $\mathrm{m}$ providing $\approx 70$ emerged seedlings $/ \mathrm{m}$. At harvest, there were $\approx 50$ to 55 roots $/ \mathrm{m}$. Each

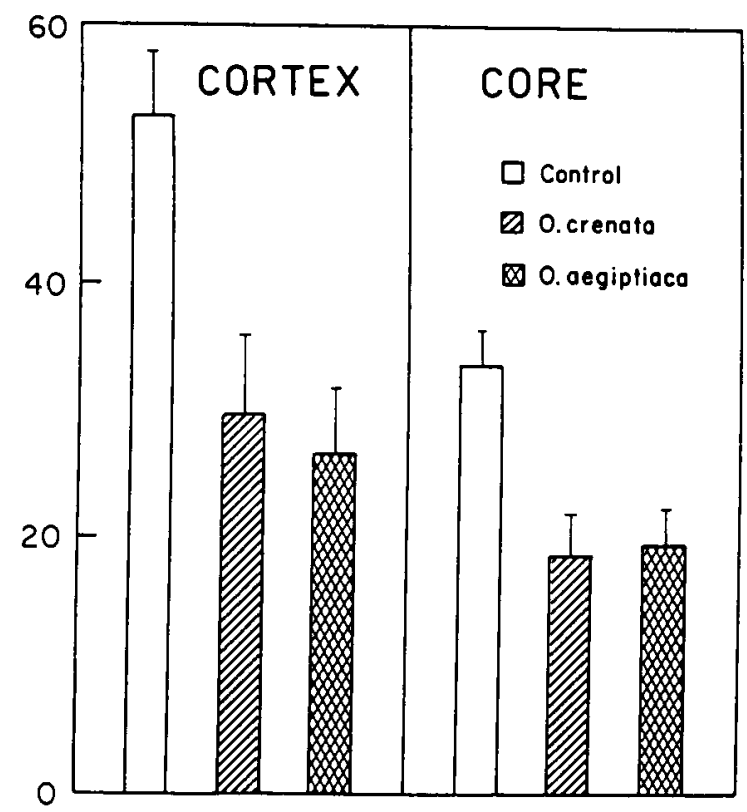

Fig. 1. Effect of Orobanche crenata and O. aegyptiaca infection on total soluble sugar content (sucrose + glucose + fructose) in the core and cortex of 'Ti-to' carrot roots. Means \pm SE of six individual carrot roots.

was to determine the effect of broomrape particularly roots that, by appearance, we An experimental plot at Bet Dagan, Israel, wathigated with hot methyl bromide at a 列

.

.

.

.

.

.

.

.

.




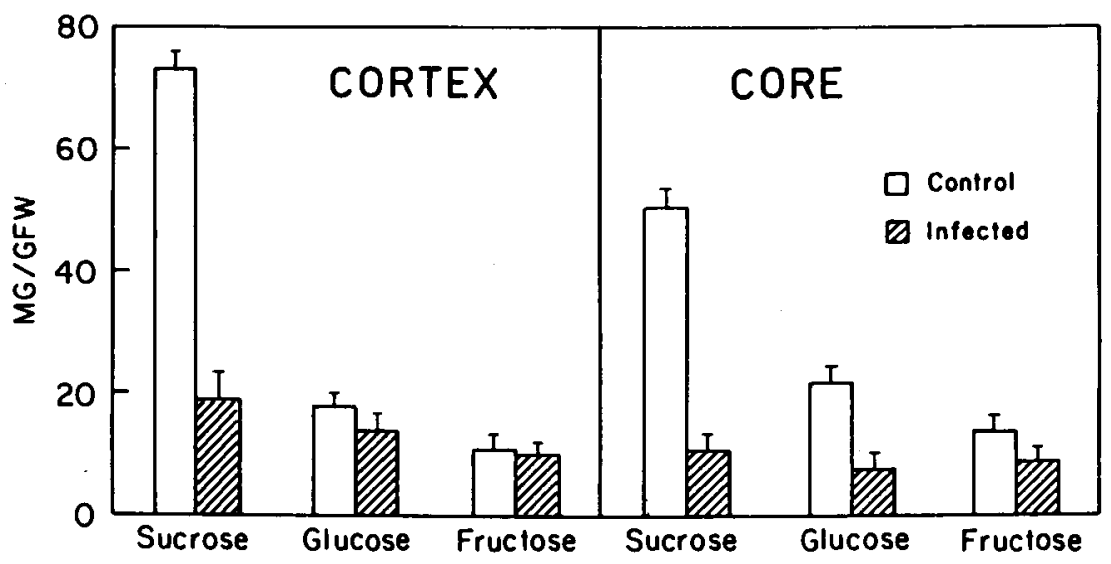

Fig. 2. Effect of Orobanche crenata infection insoluble sugar content of the core and cortex of 'Tito' carrot roots. The control and infected roots were of similar quality as judged by visual examination. Means \pm SE of six individual carrot roots.

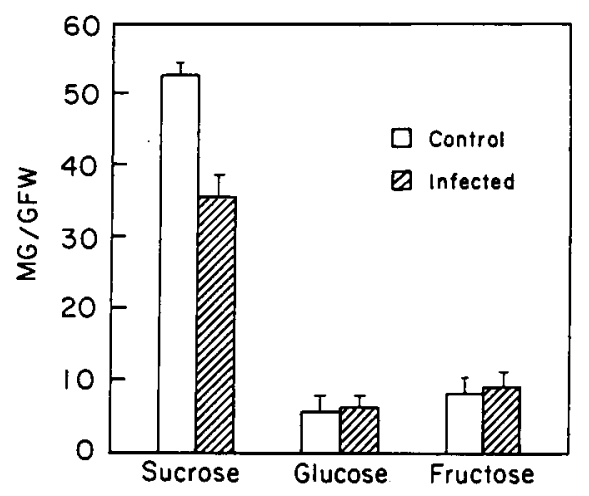

Fig. 3. Effect of Orobanche crenata field infestation on the soluble sugar content of bulk samples of 'Ti-to' carrot roots. Roots from $O$. crenata- infested plots were not individually selected for infection. Means \pm SE of six samples.

roots. Six of the infected roots were randomly sampled. In selecting the infected roots, no attempt was made to select only healthy looking roots. All root samples were separated into core and cortex.

Expt. 2. Roots were harvested on 2 May from noninfested and $O$. crenata- infested plots. Infected roots, but of normal appearance with respect to size and color, were selected. Six roots were selected from each treatment, and samples were separated into core and cortex.

Expt. 3. Three bulk samples each (25 to 30 roots) of randomly harvested roots (4 May) from control and from $O$. crenata- infested plots were brought to the laboratory. Roots of normal appearance, as above, were selected without visual selection for broomrape infection. From the roots of each of the six bulk samples, I-cm-thick cross sections, as above, were taken. From each of these six subsamples of cross sections, two samples consisting of mixed cross sections from several roots were taken. Samples were not separated into core and cortex.

$O$. aegyptiaca and $O$. crenata caused a large reduction in the total soluble sugar con- tent in the core and cortex of carrot roots (Fig. 1). There were no significant differences between the effect of the two Orobanche species studied with respect to root sugar content. Sugar content was higher in the cortex than in the core, as has been reported previously (Phan and Hsu, 1973). Starch content in both the cortex and core was low $\left(<1 \mathrm{mg} \cdot \mathrm{g}^{-1} \mathrm{fw}\right)$, as the carrot root is not a starch accumulator.

In the second experiment (Fig. 2), we compared the sugar content of noninfected roots with that of the infected but healthyappearing roots. These latter were large, wellshaped, and highly pigmented, externally indistinguishable from the noninfected carrots. The primary sugars in both infected and noninfected carrots were sucrose, glucose, and fructose, with sucrose being the predominant sugar. Sucrose content was most significantly decreased by broomrape infection in core and cortex. Infected, healthy-looking carrot roots had $\approx 30 \%$ of the sucrose content of the noninfected roots in the core and cortex.

In the third study (Fig. 3), we similarly compared the average sugar content of healthy-looking carrot roots from noninfected and infected plots. However, in this experiment no selection was made for infected roots from the infected plots. Since infected, healthy-looking roots have a greatly decreased sucrose content, we expected that bulk samples of healthy-looking carrot roots from infected plots would contain a mixture of infected and noninfected roots. Sucrose content was significantly lower in the bulk sample of carrot roots from the infested plot (Fig. 3) but not as greatly reduced as in the previous experiment, indicating that the sample presumably contained infected and noninfected roots. Accordingly, the sugar content of bulk samples should reflect the degree of infection in the field.

Orobanche crenata has been reported to withdraw sucrose from parasitized bean plants (Aber et al., 1983; Whitney, 1972). The carrot cultivar studied by us ('Ti-To') is a sucrose accumulator. It may, therefore, be suggested that Orobanche similarly removes sucrose from the carrot root. We do not know at which stage in the translocation-ur-deading-storage pathway the parasite removes the sucrose. Whitney (1972) and Aber et al. (1983) suggested that diverted sucrose was hydrolyzed in the Orobanche tubers where the hydrolysis products, glucose and fructose, are used for growth and metabolism, as well as starch synthesis and storage. Singh et al. (1968) reported high concentrations of starch in Orobanche tubers, and the Orobanche tubers we studied contained $>50 \%$ starch on a dry-weight basis (data not presented).

Our study also indicates that large amounts of sugar may be diverted or removed from storage in the carrot root without affecting easily observable signs of root quality, such as size and color. Therefore, root quality of carrots, in terms of sugar content, from fields heavily infested with broomrape can be expected to be poor, even when the plants have healthy-looking roots.

\section{Literature Cited}

Aber, M., A. Fer, and G. Sallé. 1983. Étude du transfert des substances organiques de l'hote (Vicia faba) vers le parasite (Orobanche crenata Forsk.). Z. Pflanzenphysiol. 112:297-308.

Freeman, R.E. and P.W. Simon. 1983. Evidence for simple genetic control of sugar type in carrot (Daucus carota L.). J. Amer. Soc. Hort. Sci. 108:50-54.

Jacobsohn, R., A. Greenberger, J. Katan, M. Levi, and H. Alon. 1980. Control of Egyptian broomrape (Orobanche aegyptiaca) and other weeds by means of solar heating of the soil by polyethylene mulching. Weed Sci. 28:312-316.

Joel, D.M. 1987. Detection and identification of Orobanche seeds using fluorescence microscopy. Seed Sci. Technol. 15:118-124.

Musselman, L.J. 1980. The biology of Striga, Orobanche, and other parasitic weeds. Annu. Rev. Phytopath. 18:463-489.

Musselman, L.J. 1986. Taxonomy of Orobanche, p. 2-10. In: S.J. ter Borg (ed.). Biology and control of Orobanche. LH/VPO Wageningen, The Netherlands.

Parker, C. 1986. Scope of the agronomic problems caused by Orobanche, p. 11-17. In: S.J. ter Borg (ed.). Biology and control of Orobanche. LH/VPO Wageningen, The Netherlands.

Phan, C.T. and H. Hsu. 1973. Physical and chemical changes occurring in the carrot root during growth. Can. J. Plant Sci. 53:629-634.

Schaffer, A.A., B. Aloni, and E. Fogelman. 1987. Sucrose metabolism and accumulation in developing fruit of Cucumis. Photochemistry 26:1883-1887.

Simon, P.W., C.E. Peterson, and R.C. Lindsay. 1980. Correlations between sensory and objective parameters of carrot flavor. Agr. Food Chem. 28:559-562.

Singh, M., D.V. Singh, P.C. Misra, K.K. Tewari, and P.S. Krishnan. 1968. Biochemical aspects of parasitism by the angiosperm parasites: starch accumulation. Physiol. Plant 21:525-538.

Whitney, P.J. 1972. The carbohydrate and water balance of beans (Vicia faba) attacked by broomrape (Orobanche crenata). Ann. Applied Biol. 70:59-66. 